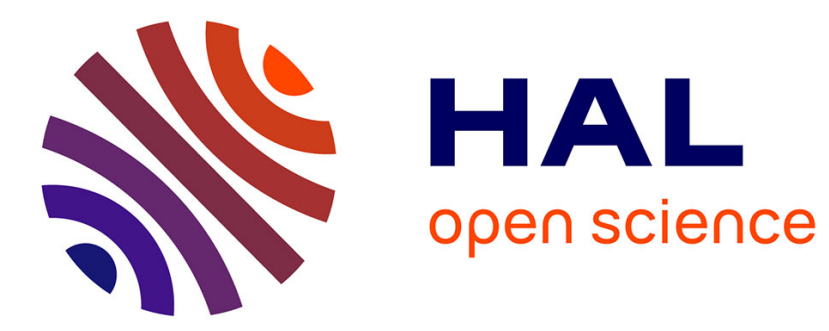

\title{
Drop generation from a vibrating nozzle in an immiscible liquid-liquid system
}

\author{
A. Bertrandias, H. Duval, Joel Casalinho, M. L. Giorgi
}

\section{To cite this version:}

A. Bertrandias, H. Duval, Joel Casalinho, M. L. Giorgi. Drop generation from a vibrating nozzle in an immiscible liquid-liquid system. Physics of Fluids, 2016, 28 (10), pp.102103. 10.1063/1.4964378 . hal-01380565

\section{HAL Id: hal-01380565 \\ https://hal.science/hal-01380565}

Submitted on 24 Oct 2016

HAL is a multi-disciplinary open access archive for the deposit and dissemination of scientific research documents, whether they are published or not. The documents may come from teaching and research institutions in France or abroad, or from public or private research centers.
L'archive ouverte pluridisciplinaire HAL, est destinée au dépôt et à la diffusion de documents scientifiques de niveau recherche, publiés ou non, émanant des établissements d'enseignement et de recherche français ou étrangers, des laboratoires publics ou privés. 


\title{
Drop generation from a vibrating nozzle in an immiscible liquid-liquid system
}

\author{
A. Bertrandias, ${ }^{\text {a) }}$ H. Duval, ${ }^{\text {b) }}$ J. Casalinho and M. L. Giorgi \\ Laboratoire de Génie des Procédés et Matériaux (LGPM), CentraleSupélec, Université Paris Saclay \\ Grande voie des vignes, 92295 Châtenay-Malabry, France
}

(Received and accepted dates, other relevant dates)

Drop generation from an axially vibrating nozzle exhibits a transition in drop diameter when varying the vibration amplitude. Below a threshold amplitude, forcing has essentially no effect on drop size and drops form in dripping mode. Above the threshold, drop size is controlled by forcing: drops detach at resonance, i.e., when the first eigenfrequency of the growing drop coincides with the forcing frequency. We experimentally study the impact of the nozzle inside diameter, dispersed phase flow rate, interfacial tension and dispersed phase viscosity on this transition. Drop diameter is well correlated to the mode 1 eigenfrequency of Strani and Sabetta for a drop in partial contact with a spherical bowl. We propose a transient model to describe drop dynamics until detachment. The drop is modelled as a linearly forced harmonic oscillator, with the eigenfrequency of Strani and Sabetta. Since the dispersed phase does not wet the nozzle tip, an additional damping coefficient is introduced to account for the viscous dissipation in the film of continuous phase between the drop and nozzle surface. The model adequately reproduces the effect of the different parameters on the threshold amplitude.

\section{INTRODUCTION}

Inducing vibration to jets or drops can be used to control breakup, thus drop size. Vibration is applied for example in ink jet printing, spray coating or vibrating cross-flow membrane emulsification, the latter having motivated our research. We focus on transversal vibrations, where drops undergo axial oscillations. For a membrane with a mean pore diameter of $0.8 \mu \mathrm{m}$, Arnaud ${ }^{1}$ found a decrease in the peak of the volume-weighted drop size distribution (from $30 \mu \mathrm{m}$ to $10 \mu \mathrm{m}$ ) at a forcing frequency of 15 to $20 \mathrm{kHz}$ compared to without vibration. Thus, vibrating the membrane in this process impacts drop size but mechanisms for drop detachment were not explained. ${ }^{1,2}$ To obtain a fine control on drop size, understanding the physics of drop vibration and detachment is necessary.

Oscillations of liquid drops have been extensively studied since the pioneering work of Lord Rayleigh $^{3}$. He calculated the eigenmodes of a free inviscid, incompressible drop in a vacuum, in absence of gravity and for small-amplitude oscillations. The eigenmodes are characterized by two integers: a polar wavenumber $n \geq 2$ and an azimuthal wavenumber $m \in[-n ; n]$. In this study, we focus on axisymmetric modes $(m=0)$. Rayleigh ${ }^{3}$ showed that the eigenfrequencies depend on $n$, the liquid density, interfacial tension and drop size. Drop eigenfrequencies scale as $D_{d}^{-3 / 2}$, with $D_{d}$ the drop diameter. Lamb ${ }^{4}$ generalized this theory by calculating the eigenpulsation of a drop in a surrounding fluid and found the same relationship $f \sim D_{d}^{-3 / 2}$.

Rodot et al. ${ }^{5}$ and Bisch et al. ${ }^{6}$ investigated drops partially bound to a rod, submitted to controlled vibration. The drop was immersed in immiscible liquids of equal density, with its contact line pinned on the rod edge. A large range of liquid couples were examined and the first eigenfrequency was found to depend on the support diameter, drop diameter, drop density and surface tension. The first resonance frequency scales as $D_{d}^{-2}$ and not as $D_{d}^{-3 / 2}$ such as for the free drop. Then, Strani and Sabetta $^{7}$ (hereafter denoted S\&S) studied linear oscillations of a liquid drop in an outer fluid, in partial contact with a spherical bowl under inviscid and zero-gravity assumptions. The presence of the support increases the eigenfrequencies for modes $n \geq 2$, but an additional low-frequency mode appears. This is the $n=1$ eigenmode, associated with the displacement of the bound drop center of mass. When the support reduces to a single point, the $n=1$ mode degenerates to a zero-frequency

\footnotetext{
a) Electronic mail: aude.bertrandias@centralesupelec.fr

b) Electronic mail: herve.duval@centralesupelec.fr
} 
rigid motion of the drop. $\mathrm{S} \& \mathrm{~S}^{7}$ noted that the mode 1 eigenfrequency may be approximated over intervals by $D_{d}^{\alpha}$ with $\alpha$ varying between -2.9 and -1.75 for drop to support diameter ratios of 1.3 to 7 , respectively. This is consistent with $\alpha=-2$ proposed by Bisch et al. ${ }^{6}$ Also, S\&S $\mathrm{S}^{7}$ computed frequencies were in agreement with Bisch et al. ${ }^{6}$ data, but resonance frequencies were overpredicted by $20 \%$ (reduced to $10 \%$ by accounting for viscous effects ${ }^{8}$ ). However, both models (inviscid ${ }^{7}$ and viscous $^{8}$ ) overpredicted resonance frequencies for large support to drop diameter ratios, attributed to nonlinear effects, not taken into account in the models. Smithwick and Boulet ${ }^{9}$ studied the first resonance frequency of mercury drops on glass (pinned contact line) under partial vacuum and compared their data to the calculations of S\&S. ${ }^{7}$ A maximum error of $3.3 \%$ was found.

Bostwick and Steen ${ }^{10}$ and Vejrazka et al. ${ }^{11}$ studied linear oscillations of a drop supported on a ring. Bostwick and Steen ${ }^{10}$ noted that the center of mass motion is partitioned among all the eigenmodes but the $n=1$ mode is its main carrier. Vejrazka et al. ${ }^{11}$ found that for small support to drop diameter ratios, the frequency response of the drop is independent of the constraint (bowl or ring). Abi Chebel et al. ${ }^{12}$ and Vejrazka et al. ${ }^{11}$ examined drop oscillations driven by imposed periodic volume variations. The frequency response is independent of the forcing type as long as the support to drop diameter ratio is small. ${ }^{11}$ Lastly, Noblin et al. ${ }^{13}$ studied bound drop oscillations with mobile instead of pinned contact lines: a decrease in resonance frequency was found. The transition from a pinned to mobile contact line occurred above a critical forcing amplitude. In that case, the variation of the contact angle exceeds the contact angle hysteresis.

Previous studies explored linear oscillations. Wilkes and Basaran ${ }^{14}$ (hereafter denoted W\&B) used computational fluid dynamics (CFD) to study large-amplitude axisymmetric oscillations of a viscous bound drop on a rod (pinned contact line). They found that the drop resonance frequency varies slightly with amplitude at high Ohnesorge numbers (Oh, expressed in section V.B.) but decreases significantly with amplitude at low $\mathrm{Oh}$, Oh being the ratio of a viscocapillary to an inertial-capillary timescale. Resonance frequency also decreases as the Bond number (Bo, expressed in section II.C.) increases. Bo compares the gravity to capillary forces. The maximum drop deformation, observed at resonance, increases with forcing amplitude and Bo and decreases with Oh and $n$. DePaoli et al. ${ }^{15}$ experimentally studied pendant drops in air under high-amplitude forcing and observed hysteresis, characteristic of soft nonlinearities. At a set forcing amplitude (resp. frequency), a larger response amplitude appeared at lower frequencies (resp. amplitudes) when a downwards frequency (resp. amplitude) sweep was performed $v$ s. an upwards sweep. $W \& \mathrm{~B}^{16}$ numerically gave the critical forcing amplitude for the onset of hysteresis for different $\mathrm{Oh}$. This value could be as low as $3 \%$ of the rod radius (drop and rod radii of the same order). Calculations were also performed for drops hanging from a tube: the first resonance frequency is slightly higher when the support is a tube, the hysteresis range is shifted to higher values of forcing frequency and the deformation at resonance is higher.

For high enough forcing amplitudes, drops detach from the support. W\&B ${ }^{17}$ used CFD to simulate drop ejection from a rod (pinned contact line). Above a critical amplitude, the bound drop ruptures: a primary drop is ejected from the liquid remaining on the rod. The variations of the critical amplitude as a function of the forcing pulsation has a $\mathrm{V}$-shape (the minimum corresponds to drop resonance). For a set rod diameter, the critical amplitude increases when Oh decreases or when the bound drop volume decreases. Critical amplitudes range from $25 \%$ to $80 \%$ of the rod radius. Kim ${ }^{18}$ experimentally studied the detachment of a pendant drop from a smooth vibrating plate in air (mobile contact line). The variations of the critical amplitude as a function of the forcing frequency has a Wshape. Kim ${ }^{18}$ found that the minima concord well with the $n=1$ and $n=2$ modes of the bound drop as calculated by S\&S. ${ }^{7}$ Again, the minima correspond to drop resonance. The agreement between data and calculations of $\mathrm{S} \& \mathrm{~S}^{7}$ is remarkable as the contact line mobility is different and experimental oscillation amplitudes are beyond the linear regime.

Resonance also triggered drop detachment in previous work of the authors, where different pore diameters were studied for one system (dodecane-water without surfactant). ${ }^{19}$ Drops were formed through a vibrating nozzle continuously fed with dodecane, immersed in the stationary immiscible water phase. This enabled to gain insight into transversally vibrating membrane emulsification in a simplified configuration. We found that at a set forcing frequency, smaller drops were generated above a threshold forcing amplitude: a growing drop detached prematurely when its first resonance frequency (as given by Bisch et al.) and the forcing frequency coincided. However, the threshold was 
higher than expected, attributed to the fact that the bound drop did not spend enough time in the resonance range to reach steady-state resonance. The generation mode forming the smaller drops was named the "stretching mode". Below the threshold, larger drops were formed in dripping mode.

The aim of this work is to study the mutual effect of forcing parameters and system properties on drop generation from a vibrating nozzle. We also aim to further model drop generation modes by accounting for drop growth and motion as a function of time. We emphasize that studies on vibrated growing drops are rare ${ }^{19}$ compared to those on constant-volume drops ${ }^{5-18}$ and that previous work concerned only one system and parameter. ${ }^{19}$ In the following, we first describe our setup. We present the dripping to stretching transition and propose a simple framework to approach it. Then, we discuss the effect of nozzle inside diameter, dispersed phase flow rate, interfacial tension and dispersed phase viscosity on the transition. We examine the effect of these parameters on (i) the threshold amplitude for the stretching mode and (ii) the resulting drop diameters. We further analyze our results by comparing them to S\&S calculations. ${ }^{7}$ Finally, we propose a simple transient model to describe drop dynamics until detachment and compare the model predictions to experiments.

\section{EXPERIMENTAL}

A. MATERIALS

The reference continuous and dispersed phases are distilled water and dodecane $(99 \%$, Fisher Scientific), respectively. To study the impact of interfacial tension, a surfactant (SDS, $85 \%$, Acros Organics) is added to the continuous phase at $0.1 \mathrm{wt} \%$ or $2 \mathrm{wt} \%$ (systems 1 and 2, resp.). To study the impact of dispersed phase viscosity, paraffin (Fisher Scientific) is added to the dispersed phase at 25 wt $\%$ or $50 \mathrm{wt} \%$ (systems 3 and 4, resp.). A system with an increased continuous phase viscosity was also tested (supplementary material D). The reference system and system 1 to 4 properties are given in table I. The viscosities $\eta$ and densities $\rho$ of the mixtures were measured in triplicate, the former with a Ubbelohde type viscosimeter (AVS310, Schött-Gerade) at $25.1^{\circ} \mathrm{C}$. The interfacial tension $\gamma$ was measured in triplicate by the rising drop method with a tensiometer (Tracker, I.T. Concept, Teclis). For systems 1 and 2, $\gamma$ is determined by the method explained in the supplementary material A. Table I values report an intermediate plateau interfacial tension. We consider that the plateau value gives an adequate estimation of the interfacial tension when drops form (see supplementary material B).

Table I. Properties of the different systems investigated.

\begin{tabular}{|c|c|c|c|c|c|c|c|}
\hline System & Dispersed phase 'dp' & $\begin{array}{c}\eta_{d p} \\
\text { (mPa.s) }\end{array}$ & $\begin{array}{c}\rho_{d p} \\
\left(\mathrm{~kg} \cdot \mathrm{m}^{-3}\right)\end{array}$ & $\begin{array}{c}\text { Continuous phase } \\
\text { 'cp' }\end{array}$ & $\begin{array}{c}\eta_{c p} \\
\text { (mPa.s) }\end{array}$ & $\begin{array}{c}\rho_{c p} \\
\left(\mathrm{~kg} \cdot \mathrm{m}^{-3}\right)\end{array}$ & $\gamma\left(\mathrm{mN} \cdot \mathrm{m}^{-1}\right)$ \\
\hline Reference & & 1.34 & 750 & Distilled water & 0.89 & 997 & $50.7 \pm 3.5^{\mathrm{a}}$ \\
\hline 1 & & 1.34 & 750 & $\begin{array}{l}\text { Distilled water and } \\
\text { SDS }(0.1 \mathrm{wt} \%)\end{array}$ & 0.89 & $\begin{array}{c}997 \pm \\
1.4^{\mathrm{a}}\end{array}$ & $19.0 \pm 0.6^{\mathrm{a}, \mathrm{b}}$ \\
\hline 2 & & 1.34 & 750 & $\begin{array}{l}\text { Distilled water and } \\
\text { SDS }(2 \mathrm{wt} \%)\end{array}$ & 0.89 & $\begin{array}{c}1001 \pm \\
1.2^{\mathrm{a}}\end{array}$ & $5.4 \pm 0.5^{\mathrm{a}, \mathrm{b}}$ \\
\hline 3 & $\%)$ & $\begin{array}{l}1.79 \pm \\
0.23^{\mathrm{a}}\end{array}$ & $\begin{array}{c}772 \pm \\
1.4^{\mathrm{a}}\end{array}$ & Distilled water & 0.89 & 997 & $53.5 \pm 2.4^{\mathrm{a}}$ \\
\hline & $\begin{array}{l}0 \mathrm{wt} \%) \\
50 \mathrm{wt} \%)\end{array}$ & $\begin{array}{l}3.24 \pm \\
0.42^{\mathrm{a}}\end{array}$ & $\begin{array}{c}790 \pm \\
1.1^{\mathrm{a}}\end{array}$ & Distilled water & 0.89 & 997 & $50.0 \pm 1.2^{\mathrm{a}}$ \\
\hline
\end{tabular}

${ }^{a}$ the tabulated value is measured experimentally.

${ }^{b}$ the tabulated value corresponds to the dynamic interfacial tension measured at the intermediate plateau.

\section{B. EXPERIMENTAL SETUP}

The setup, illustrated in prior work $^{19}$, is summarized in fig. 1. A single glass capillary (nozzle) of inside diameter $D_{p}$ emerges into a tank with the stationary continuous phase. Two pore diameters are presently tested: $D_{p}=0.32 \mathrm{~mm}$ and $D_{p}=0.11 \mathrm{~mm}$. The dispersed phase is supplied through the pore at a flow rate $q=1.1 \mu \mathrm{L} . \mathrm{s}^{-1}$ to $14.4 \mu \mathrm{L} . \mathrm{s}^{-1}$ (PHD Ultra Syringe Pump, Harvard Apparatus), leading to mean flow velocities $v_{d p}=4 q /\left(\pi D_{p}^{2}\right)$. Reynolds numbers for the flow in the nozzle are of $\operatorname{Re}_{\text {cap }}=$ 3.0 to $32.8\left(\mathrm{Re}_{\text {cap }}=\rho_{d p} v_{d p} D_{p} / \eta_{d p}\right)$ (laminar flow). The nozzle is fixed on a vibrating exciter (Bruel $\&$ Kjaer 4810) which induces a sinusoidal motion $x_{\text {cap }}$ in time $t: x_{\text {cap }}=A \sin (2 \pi f t) . A$ is the forcing amplitude measured by a laser sensor (M5L/2, Bullier Automation) with a precision in the 
order of $10 \mu \mathrm{m} . f$ is the forcing frequency set on the signal generator (33512B Arbitrary Waveform Generator, Agilent). Vibrations are parallel to the nozzle axis, so drops undergo axial oscillations.

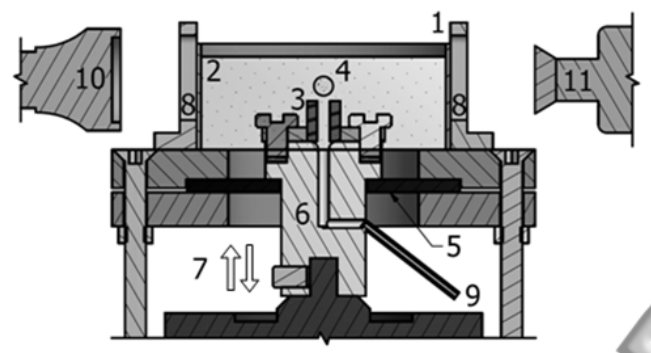

FIG. 1. Cross-section side view of the setup by CAD: 1, tank; 2, continuous phase; 3, glass capillary (nozzle); 4, dispersed phase drop; 5 , flexible seal; 6 , central element fixed on the vibrating exciter; 7, axial, vibrating motion; 8, windows; 9 , dispersed phase supply system; 10, light source; 11, high-speed camera with macro lens.

In the moving non-inertial frame of reference where the nozzle is still (axes in fig. 2), the forces exerted on the drop due to nozzle motion are the inertial force and associated Archimedes' thrust. We note that the continuous phase above the nozzle and support is accelerated by the exciter, shown by Faraday waves at the free surface. The resulting excitation force is:

$$
F_{\text {exc }}=\left(\rho_{c p}-\rho_{d p}\right) \frac{\pi}{6} D_{d}^{3} a_{\text {cap }}
$$

with $D_{d}$ the bound drop diameter and $a_{\text {cap }}=A \omega^{2} \sin (\omega t+\pi)$ the nozzle acceleration in the laboratory inertial frame ( $\omega=2 \pi f$ is the forcing pulsation). Drop formation is recorded with a highspeed camera (v310, Phantom) and macro lens (AF Zoom-Micro Nikkor 70-180mm f/4.5-5.6D ED, Nikon). The acquisition frequency is ten times the forcing frequency or $100 \mathrm{fps}$ for trials without vibration. The resolution is $800 \times 600 \mathrm{px}^{2}$. We extract data with Image $\mathrm{J}^{20}$ including average detached drop diameters $D_{d}$, axial drop elongations $L$ and the position of the drop center of mass $X_{d}$ compared to the nozzle surface. Images were calibrated $(36 \mathrm{px} / \mathrm{mm})$ using the outer diameter $(7.86 \pm 0.01 \mathrm{~mm}$ for $D_{p}=0.32 \mathrm{~mm}$ ) of the nozzle. The main output data are resumed in fig. 2 .

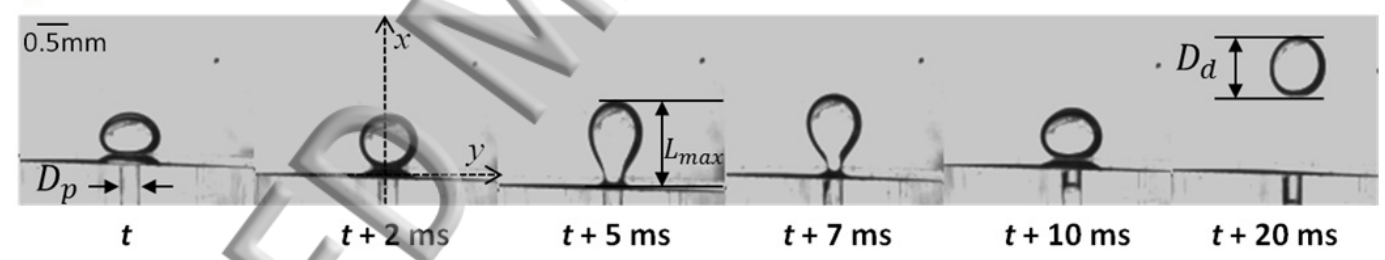

FIG. 2. Visual summary of the output data. Drop detaching in stretching mode in time $t$ for the reference system, $\boldsymbol{D}_{\boldsymbol{p}}=0.32$ $\mathrm{mm}, \boldsymbol{q}=3.6 \mu \mathrm{L} \cdot \mathrm{s}^{-1}, \boldsymbol{f}=100 \mathrm{~Hz}, \boldsymbol{A}=0.209 \mathrm{~mm}$.

\section{EXPERIMENTAL PROTOCOL}

The tank is filled with the continuous phase and the tube and syringe with the dispersed phase. The syringe pump is activated and drop diameters are measured without vibration. Drops are formed in dripping mode. We calculate Bond numbers Bo and Weber numbers We as Clanet and Lasheras ${ }^{21}$ : we find $\mathrm{Bo}=\left[\left(\rho_{c p}-\rho_{d p}\right) g D_{p}^{2} / 2 \gamma\right]^{1 / 2}$ (with $g$ the gravitational acceleration) from $1.7 \times 10^{-2}$ to $1.5 \times 10^{-1}$ and $\mathrm{We}=\rho_{d p} v_{d p}^{2} D_{p} / \gamma$ from $4.5 \times 10^{-3}$ to $2.8 \times 10^{-1}$. These values are below the critical We for the transition to jetting (at the given Bo), confirming the setup operates in dripping mode. From the drop diameters obtained without vibration, we calculate the in situ interfacial tensions by Tate's law ${ }^{22}$ :

$$
\left(\rho_{c p}-\rho_{d p}\right) \frac{\pi}{6} D_{d}^{3} g=\mathcal{F}_{H B} \pi D_{p} \gamma
$$

Drop detachment occurs when buoyancy (left-hand side of Eq. (2)) exceeds the maximum capillary force $F_{\gamma}^{\max }=\pi D_{p} \gamma$ that the drop neck can resist without breaking. $D_{d}$ is the detached drop diameter. $\mathcal{F}_{H B}$ is the Harkins Brown correction factor ${ }^{23}$ : it accounts for the fraction of liquid volume which stays attached to the nozzle after drop detachment. We use the $\mathcal{F}_{H B}$ factor of Mori. ${ }^{24}$ The in situ interfacial tension is then compared to the measured one (table I), to ensure the setup is adequately cleaned. 
Then, vibration is applied. A forcing frequency is set and an upwards amplitude sweep is performed. Measurements are made at different amplitudes, ensuring one is always made at the threshold where a transition in drop generation occurs (see III.). This is repeated for frequencies from 30 to $150 \mathrm{~Hz}$. From 30 to $100 \mathrm{~Hz}, 10 \mathrm{~Hz}$ intervals are applied. Above $100 \mathrm{~Hz}$, intervals vary depending on the pore diameter. The vibrating exciter limitations do not enable us to observe the stretching mode above $150 \mathrm{~Hz}$ for $D_{p}=0.32 \mathrm{~mm}$ and $110 \mathrm{~Hz}$ for $D_{p}=0.11 \mathrm{~mm}$. After trials, a cleaning agent at $3 \mathrm{vol} \%$ (Mucasol, Merz) fills the setup for $24 \mathrm{~h}$ and it is rinsed with distilled water leading to a hydrophilic glass surface. Then, the organic dispersed phase does not wet the nozzle and the outer nozzle diameter does not influence drop detachment.

For each test condition (i.e., physicochemical system, pore diameter, dispersed phase flow rate and forcing frequency), three trials are carried out to determine the transition threshold. For each trial, six detached drops are studied. For each drop, ten images are analyzed. We checked that the accuracy of the diameter measurement from ten different snapshots of a given drop is sub-pixel. For a given trial, we noted variations up to $2 \mathrm{px}$ at most in diameter from one drop to another. In the figures displaying drop diameters, the error bars correspond to the relative standard deviation in drop diameters: it ranges between $1 \%$ to $7 \%$ depending on the test conditions.

\section{TRANSITION FROM DRIPPING TO STRETCHING MODE}

Figure 3 shows typical variations of the drop diameter as a function of the forcing amplitude at a set forcing frequency $f$. The drop diameter falls (by $63 \%$ ) at a threshold amplitude $A_{t h}$. The same behavior occurs for all systems. For the reference system, a relative decrease in drop diameter of $45 \%$ to $76 \%$ was found at $A_{t h}$ compared to without vibration depending on $f$, for all pore diameters. For system 2, similar values were found: $29 \%$ to $73 \%$. This fall at $A_{t h}$ corresponds to a transition in the drop generation regime. For $A<A_{t h}$, drops detach in dripping mode and their diameter is close to the diameter of the drop formed without vibration: detachment is buoyancy-controlled. For $A>A_{t h}$, the drop detaches when its mode 1 eigenfrequency coincides with $f$ and when it reaches a critical elongation ratio: detachment is controlled by the excitation force. Figure 2 shows how a drop elongates at resonance (characteristic mode 1 resonance shape) and detaches. We named this the "stretching mode". ${ }^{19}$ It should be noted that there is an amplitude interval where both modes coexist. The threshold amplitude $A_{t h}$ is defined as the upper bound of that interval, when all drops are generated in stretching mode.

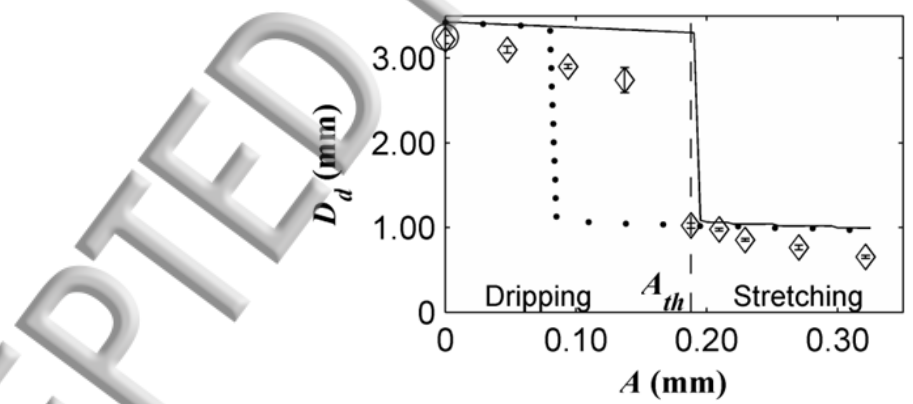

FIG. 3. Transition from dripping to stretching mode: generated drop diameter as a function of the forcing amplitude for the reference system, $\boldsymbol{D}_{p}=0.32 \mathrm{~mm}, \boldsymbol{f}=100 \mathrm{~Hz}, \boldsymbol{q}=3.6 \mu \mathrm{L} . \mathrm{s}^{-1}$. ( $\diamond$ ) Experimental data; experimental threshold (dashed line); (O) theoretical drop size without vibration from Eq. (2); simulation results of section V.C., Eq. (14) (dotted line); of section V.D., Eq. (17) with $\boldsymbol{\alpha}=-1.9, \boldsymbol{C}=4.4$ (solid line).

We proposed a simple model ${ }^{19}$ to describe the main features of stretching mode. Below, we recall its main arguments and derive scaling laws to provide a framework to analyze our experimental results in section IV. The vibrating bound drop is considered as a linearly forced harmonic oscillator (LFHO) with moderate damping. ${ }^{25}$ Drop growth is considered slow enough for oscillations to reach steady state. We are aware that these assumptions are strong since the system is probably no longer linear when oscillations are such that the drop detaches and the process remains transient. However they are required to develop the following scaling laws.

A simple analytical expression of the mode 1 eigenfrequency $f_{1}$ of a bound drop has been empirically established by Bisch et al. ${ }^{6}$ for $D_{d} / D_{p}$ of 1.3 to 7 and fluids of equal densities: 


$$
f_{1}=\frac{1}{2 \pi} \sqrt{\frac{6 K \gamma}{\pi \rho_{d p}}} \frac{\sqrt{D_{p}}}{D_{d}^{2}}
$$

$K$ is a constant that should depend on the fluid density ratio, with $K=9$ for fluids of equal densities. $D_{d}$ is the resonating bound drop diameter, which we assimilate to the detached drop diameter.

Bisch et $a l .^{6}$ also propose an empirical expression for the damping coefficient $\beta$ :

$$
\beta / f_{1}=a\left(\eta_{d p} / \rho_{d p}\right)+b\left(\eta_{c p} / \rho_{c p}\right)
$$

with $a=4.5 \times 10^{3}$ and $b=2.32 \times 10^{5}$ if $\eta_{d p} / \rho_{d p}>\eta_{c p} / \rho_{c p}$ and $a=1.57 \times 10^{5}$ and $b=4.5 \times$ $10^{4}$ if $\eta_{d p} / \rho_{d p}<\eta_{c p} / \rho_{c p}$. We assume Eq. (3) and (4) can be reasonably applied to our trials as the density ratio $\rho_{c p} / \rho_{d p}$ is in the order of 1 (it ranges from 1.26 to 1.33 ). Also, we neglect the effect of buoyancy on $f_{1}$ and $\beta$.

From Eq. (2) and (3), omitting $\mathcal{F}_{H B}$, we deduce the minimum forcing frequency above which a growing drop may detach in stretching mode (if $A>A_{t h}$ ):

$$
f_{1, \text { min }}=\frac{K^{1 / 2}}{2 \times 6^{1 / 6} \pi^{3 / 2}} \frac{\left|\rho_{c p}-\rho_{d p}\right|^{2 / 3} g^{2 / 3}}{\gamma^{1 / 6} D_{p}^{1 / 6} \rho_{d p}^{1 / 2}}
$$

The minimum forcing frequency is around $8 \mathrm{~Hz}$ for $D_{p}=0.32 \mathrm{~mm}$ for the reference system and 14 $\mathrm{Hz}$ for $D_{p}=0.11 \mathrm{~mm}$ for system 2 . This is smaller than the lower bound of the frequency range investigated. Consequently, drops may detach in stretching mode.

Whatever the mode (dripping or stretching), the drop detaches when the restoring capillary force $F_{\gamma}$ exceeds the maximum capillary force $F_{\gamma}^{\max }$. Under LFHO assumption, $F_{\gamma}$ reads:

$$
F_{\gamma}=\frac{\pi}{6} D_{d}^{3} \rho_{d p} \omega_{1}^{2} x_{d}(t)
$$

with $\omega_{1}$ the eigenpulsation of the bound drop without damping. $x_{d}$ is the displacement of the drop center of mass with respect to its rest position (absence of buoyancy and excitation forces). The forcebased detachment criterion can be easily recast into an elongation-based criterion:

$$
x_{d}(t) \geq \frac{6 D_{p} \gamma}{D_{d}^{3} \rho_{d p} \omega_{1}^{2}}
$$

The displacement of the drop center of mass $x_{d}$ is made up of a stationary part due to buoyancy and an oscillatory part due to the excitation force. Assuming quasi steady state, $x_{d}$ reads:

$$
x_{d}(t)=\frac{\phi g}{\omega_{1}^{2}}+A_{d} \sin (\omega t+\delta)
$$

with $\phi=\left(\rho_{c p}-\rho_{d p}\right) / \rho_{d p}, \delta$ the phase shift and $A_{d}$ the amplitude given by the well-known expression $^{25}$ :

$$
A_{d}=\frac{\omega^{2} \phi A}{\sqrt{\left(\omega_{1}^{2}-\omega^{2}\right)^{2}+Q^{-2} \omega_{1}^{2} \omega^{2}}}
$$

$Q$ is the quality factor given by $Q=\omega_{1} /(2 \beta)$. Since $\beta$ is given by Eq. (4), $Q$ depends only on the phase densities and viscosities. $A_{d}$ is maximum when $\omega_{1}\left(D_{d}\right)=\omega\left(1-1 /\left(2 Q^{2}\right)\right)^{1 / 2}$, i.e., $\omega_{1}\left(D_{d}\right) \cong$ $\omega$ for moderate damping. In that case, $A_{d}$ simplifies to $A_{d} \cong Q \phi A$.

In dripping mode, the drop has left the resonance range and its eigenfrequency is much lower than the forcing frequency. Then, buoyancy dominates: Eq. (7) and (8) reduce to Eq. (2) (omitting $\mathcal{F}_{H B}$ ). In stretching mode, the drop detaches at resonance $\left(\omega_{1} \cong \omega\right)$. Its diameter is thus:

$$
D_{d} \cong\left(\frac{6 K \gamma D_{p}}{\pi \rho_{d p}}\right)^{1 / 4} \frac{1}{\sqrt{\omega}}
$$


An estimate of the threshold amplitude may be derived from the above equations neglecting buoyancy and assuming that the detachment criterion is satisfied at an oscillation peak:

$$
A_{t h} \cong\left(\frac{\pi}{K}\right)^{3 / 4}\left(\frac{6 D_{p} \gamma}{\rho_{d p}}\right)^{1 / 4} \frac{1}{Q \phi \sqrt{\omega}}
$$

As a result, $D_{d}$ and $A_{t h}$ should both scale as $\omega^{-1 / 2}$, i.e., $f^{-1 / 2}$.

\section{IMPACT OF PROCESS PARAMETERS AND SYSTEM PROPERTIES}

In this section, we study the effect of process parameters (pore diameter, dispersed phase flow rate) and system properties (interfacial tension, dispersed phase viscosity) on the dripping to stretching transition. Threshold amplitudes $A_{t h}$ are determined from an amplitude sweep and drop diameters at $A_{t h}$ from image analysis. Error bars are generally large for threshold amplitudes partly due to measurement errors and partly due to the difficulty to repeatedly estimate the threshold.

\section{A. INFLUENCE OF PORE DIAMETER}

Fig. 4 reports the variations of the threshold amplitude and generated drop diameter as a function of the forcing frequency for two pore diameters, i.e., $D_{p}=0.11 \mathrm{~mm}$ and $0.32 \mathrm{~mm}$ (more pore diameters were tested in another paper in the case of the reference system ${ }^{19}$ ). Threshold amplitude variations with forcing frequency are monotonous (fig. 4(a)) and do not exhibit the V-or W-shape

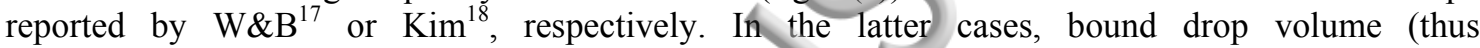
eigenfrequencies) are fixed, independently of $f$. On the contrary, in our setup, the drop grows until its eigenfrequency coincides with $f$.

The threshold amplitude decreases as the forcing frequency increases (fig. 4(a)), in accordance with Eq. (11). Eq. (11) predicts a $f^{-1 / 2}$ scaling but our data scale differently: $f^{-0.71 \pm 0.07}$ for $D_{p}=$ $0.11 \mathrm{~mm}$ and $f^{-1.05 \pm 0.13}$ for $D_{p}=0.32 \mathrm{~mm}$. Also, thresholds are twice higher for $D_{p}=0.11 \mathrm{~mm}$ than for $D_{p}=0.32 \mathrm{~mm}$. This contradicts the expected $D_{p}^{1 / 4}$ scaling (Eq. (11)). We return to this in section $\mathrm{V}$.

The drop diameter decreases with increasing forcing frequency (fig. 4(b)). The $f^{-1 / 2}$ scaling predicted by Eq. (10) was verified for four pore diameters ranging from $0.11 \mathrm{~mm}$ to $0.75 \mathrm{~mm}^{19}$ : we specifically find $f^{-0.48 \pm 0.03}$ for $D_{p}=0.11 \mathrm{~mm}$ and $f^{-0.48 \pm 0.04}$ for $D_{p}=0.32 \mathrm{~mm}$. The larger the pore diameter, the larger the drops produced. However, from experimental data ${ }^{19}$, it is difficult to conclude on the relevance of the predicted $D_{p}^{1 / 4}$ scaling as the deviation of the data to the $D_{p}^{1 / 4}$ scaling is large $(0 \%$ to $31 \%$, depending on $f)$.
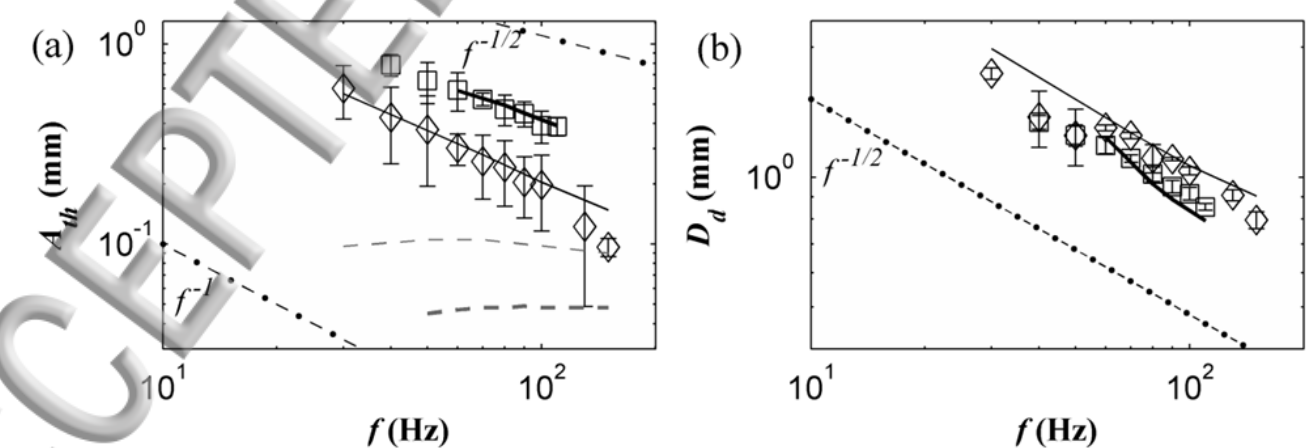

FIG. 4. Impact of pore size on (a) threshold amplitude $\boldsymbol{A}_{\boldsymbol{t h}}$ and (b) drop diameter at $\boldsymbol{A}_{\boldsymbol{t} \boldsymbol{h}}$, for the reference system. ( $\square$ ) $\boldsymbol{D}_{\boldsymbol{p}}=$ $0.11 \mathrm{~mm}, \boldsymbol{q}=2.2 \mu \mathrm{L} . \mathrm{s}^{-1} ;(\diamond) \boldsymbol{D}_{\boldsymbol{p}}=0.32 \mathrm{~mm}, \boldsymbol{q}=6.1 \mu \mathrm{L} . \mathrm{s}^{-1}$. Simulations from Eq. (14) of V.C. (dashed line); Eq. (17) of V.D. (solid line): $\boldsymbol{D}_{\boldsymbol{p}}=0.11 \mathrm{~mm}$ (thick); $\boldsymbol{D}_{\boldsymbol{p}}=0.32 \mathrm{~mm}$ (thin).

\section{B. INFLUENCE OF DISPERSED PHASE FLOW RATE}

Four dispersed phase flow rates $\boldsymbol{q}$ were applied to the reference system, for $D_{p}=0.32 \mathrm{~mm}: \boldsymbol{q}=$ $2.5 \mu \mathrm{L} . \mathrm{s}^{-1}, 4.3 \mu \mathrm{L} . \mathrm{s}^{-1}, 6.5 \mu \mathrm{L} . \mathrm{s}^{-1}$ and $14.4 \mu \mathrm{L} . \mathrm{s}^{-1}$. Threshold amplitudes and drop diameters do not vary significantly with these flow rates according to the error bars (see supplementary material C). This is consistent with Eq. (10) and (11). For higher flow rates, this parameter could become significant. A 
drop may no longer have time to reach large-amplitude oscillations at resonance for stretching mode. Also, a transition to jetting would occur ${ }^{21,26,27}$ (out of the scope of this paper).

When $q$ increases from 2.5 to $14.4 \mu \mathrm{L} . \mathrm{s}^{-1}$, the mean number of oscillations between two drops at $f=100 \mathrm{~Hz}$ decreases from 28 to 4 . As the threshold is little affected by $q$ in the investigation range, we infer that the steady-state oscillation regime is reached in just a few oscillations.

\section{INFLUENCE OF INTERFACIAL TENSION}

Experiments were carried out for the reference system and systems 1 and $2\left(\gamma=50.7 \mathrm{mN} \cdot \mathrm{m}^{-1}, 19.0\right.$ $\mathrm{mN} . \mathrm{m}^{-1}$ and $5.4 \mathrm{mN} \cdot \mathrm{m}^{-1}$, resp.) for $D_{p}=0.32 \mathrm{~mm}$. The threshold amplitude scaling is not significantly affected by $\gamma: f^{-0.95 \pm 0.08}$ for system 1 and $f^{-0.95 \pm 0.26}$ for system 2 compared to $f^{-1.05 \pm 0.13}$ for the reference system (fig. 5(a)). However, it is not in accordance with the predicted $f^{-1 / 2}$ scaling (Eq. (11)). Higher SDS concentrations result in lower interfacial tensions, leading to lower threshold amplitudes for a drop to detach in stretching mode (fig. 5(a)). This is in qualitative agreement with Eq. (11) but it is difficult to conclude on the relevance of the predicted $\gamma^{1 / 4}$ scaling, as the deviation of the data to this scaling is large ( $8 \%$ to $32 \%$, depending on $f$ ).

The $f^{-1 / 2}$ scaling of the drop diameter is maintained when the interfacial tension is decreased from $50.7 \mathrm{mN} \cdot \mathrm{m}^{-1}$ to $5.4 \mathrm{mN} \cdot \mathrm{m}^{-1}$ (fig. $5(\mathrm{~b})$ ): we find $f^{-0.48 \pm 0.01}$ for system 1 and $f^{-0.49 \pm 0.02}$ for system 2 . Smaller drops are generated for lower interfacial tensions. The drop diameter roughly scales as $\gamma^{1 / 4}$ in accordance with Eq. (10) (deviations of $1 \%$ to $18 \%$, depending on $f$ ).
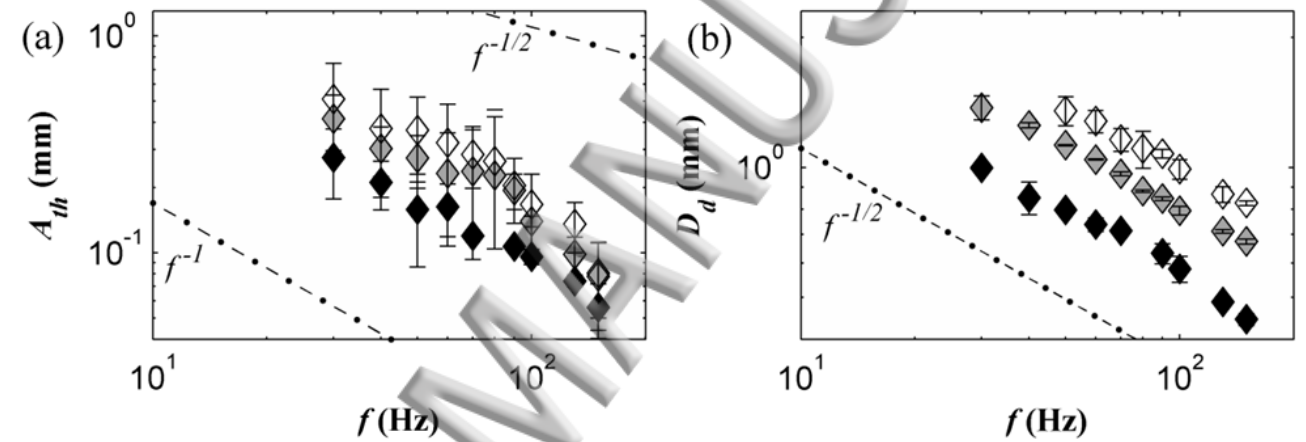

FIG. 5. Impact of interfacial tension on (a) threshold amplitude $\boldsymbol{A}_{\boldsymbol{t h}}$ and (b) drop diameter at $\boldsymbol{A}_{\boldsymbol{t} \boldsymbol{h}}$, for $\boldsymbol{D}_{\boldsymbol{p}}=0.32 \mathrm{~mm}, \boldsymbol{q}=$ $3.6 \mu \mathrm{L} . \mathrm{s}^{-1} .(\diamond)$ Reference system; $(\diamond)$ system $1 ;(\bullet)$ system 2 .

\section{INFLUENCE OF DISPERSED PHASE VISCOSITY}

Experiments were carried out for the reference system and systems 3 and $4\left(\eta_{d p}=1.34 \mathrm{mPa} . \mathrm{s}\right.$, $1.79 \mathrm{mPa} . \mathrm{s}$ and $3.24 \mathrm{mPa} . \mathrm{s}$, resp.) for $D_{p}=0.32 \mathrm{~mm}$. Threshold amplitudes $A_{t h}$ increase when $\eta_{d p}$ increases (fig. 6(a)), as in W\&B calculations. ${ }^{17}$ When changing the reference system for system 3 (resp. 4), $\eta_{d p}$ increases by $34 \%$ (resp. $142 \%$ ) and $A_{t h}$ increases by $28 \%$ to $79 \%$ (resp. $62 \%$ to $133 \%$ ). The effect of $\eta_{d p}$ is stronger than expected. Indeed, when the reference system is changed for system 4, the quality factor $Q$ decreases from 14.6 to 13.9 (Eq. (4)), leading to a theoretical 5\% increase in $A_{\text {th }}$ (Eq. (11)). In addition, the $f^{-1}$ scaling with the reference system is not conserved for systems 3 and 4 : we find $f^{-1.25 \pm 0.07}$ for system 3 and $f^{-0.73 \pm 0.12}$ for system 4 . Thus, these results do not agree with the predicted $f^{-1 / 2}$ scaling (Eq. (11)). $\eta_{d p}$ does not significantly impact drop diameter (fig. 6(b)), in agreement with Eq. (10) (valid for moderate damping).

As mentioned above, a system with a greater continuous phase viscosity was also studied: results and analysis are reported in supplementary material D. 

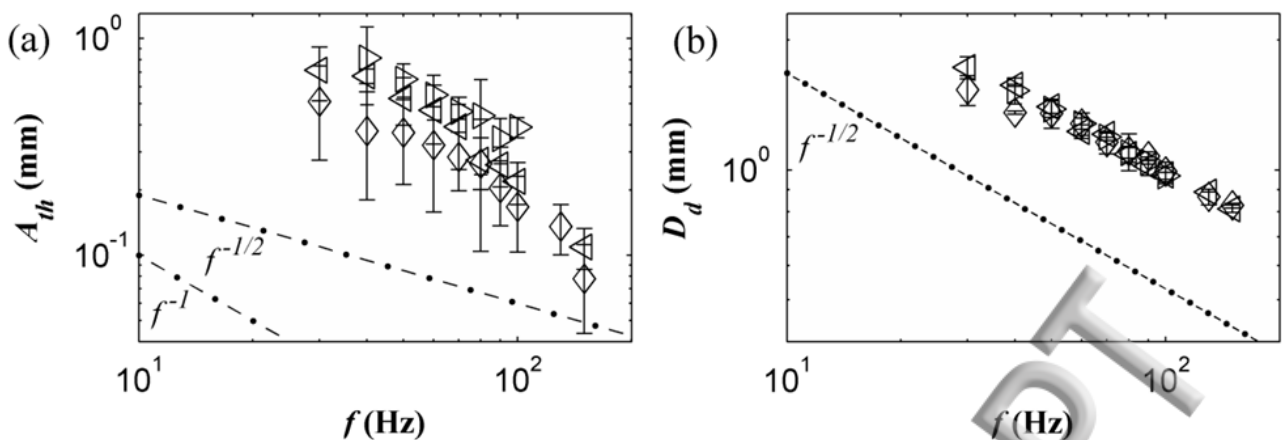

FIG. 6. Impact of dispersed phase viscosity on (a) threshold amplitude $\boldsymbol{A}_{\boldsymbol{t} \boldsymbol{h}}$ and (b) drop diameter at $\boldsymbol{A}_{\boldsymbol{t} \boldsymbol{h}}$, for $\boldsymbol{D}_{\boldsymbol{p}}=0.32 \mathrm{~mm}$, $\boldsymbol{q}=6.1 \mu \mathrm{L} \cdot \mathrm{s}^{-1} .(\diamond)$ Reference system; $(\triangleleft)$ system $3 ;(\triangleright)$ system 4.

\section{FURTHER ANALYSIS}

In this section, we synthesize drop diameter data of section IV. Then, we analyze the elongation ratio for detachment. Finally, we propose a LFHO model that better reflects the $A_{t h}$ data.

\section{A. MODE 1 RESONANCE}

Drop diameters at the threshold are consistent with the detachment at resonance when the bound drop mode 1 eigenfrequency coincides with the forcing frequency. To quantify the discrepancy between our data and Eq. (3) of Bisch et al. ${ }^{6}$, we plot the dimensionless forcing pulsation (our data) and dimensionless drop eigenpulsation (Eq. (3)) against the drop to pore diameter, for different pore diameters and interfacial tensions (fig. 7(a)) and dispersed phase viscosities (fig. 7(b)). Pulsations are made dimensionless by $\left(8 \gamma /\left(\rho_{d p} D_{d}^{3}\right)\right)^{1 / 2}$ and are labeled with a * exponent.
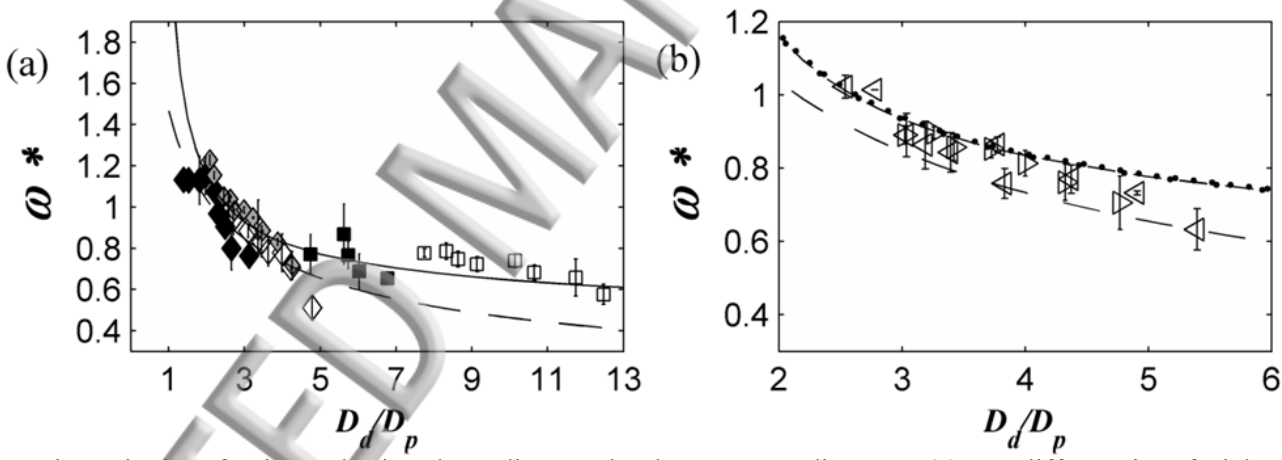

FIG. 7. Dimensionless forcing pulsation depending on the drop to pore diameter. (a) For different interfacial tensions and pore diameters: reference system (white); system 1 (grey); system 2 (black). ( $\square$ ) $\boldsymbol{D}_{\boldsymbol{p}}=0.11 \mathrm{~mm}$; ( $\left.\diamond\right) \boldsymbol{D}_{\boldsymbol{p}}=0.32 \mathrm{~mm}$. (b) For different dispersed phase viscosities, $\boldsymbol{D}_{\boldsymbol{p}}=0.32 \mathrm{~mm}$ : $(\triangleleft)$ system 3; $(\triangleright)$ system 4 . Curve of the dimensionless eigenpulsation from: Eq. (3) of Bisch et al. ${ }^{6}$ (dashed line); Eq. (12) of $\mathrm{S} \& \mathrm{~S}^{7}$ for the reference system (solid line), system 3 (dash-dotted line) and system 4 (dotted line).

Our data are well represented by Eq. (3) of Bisch et al. ${ }^{6}$ (dashed line) until $D_{d} / D_{p}=5$. For $D_{d} / D_{p}>5$, our data are markedly above the Bisch et al. ${ }^{6}$ curve. As stated, Eq. (3) was validated until $D_{d} / D_{p}=7$. As the validity of the Bisch et al. ${ }^{6}$ law is restricted, we consider the theoretical results of $\mathrm{S} \& \mathrm{~S}^{7,8}$ established for any $D_{d} / D_{p}$ and density ratio. They analyzed the axisymmetric vibrations of a liquid drop in an outer fluid, in partial contact with a spherical bowl (see fig. 8) under the assumptions of zero gravity, negligible viscous effects and small surface deformations. Their calculated eigenfrequency $f_{n}$ of mode $n$ is:

$$
f_{n}=\frac{1}{2 \pi} \sqrt{\frac{8 \gamma}{D_{d}^{3} \rho_{d p} \lambda_{n}}}
$$


with $\lambda_{n}$ the eigenvalue for mode $n$, function of the support angle $\Theta$ and phase density ratio. Assuming fig. 8 is a reasonable simplification of our drop, we estimate $\Theta=\arcsin \left(D_{p} / D_{d}\right)$. We calculate $\lambda_{1}$ with Smithwick and Boulet's method ${ }^{9}$ derived from the work of $S \& S^{7}$, using densities and interfacial tensions of table I. Our data are better fitted by the model of $S \& S^{7}$ than by the law of Bisch et al. ${ }^{6}$ (fig. 7(a) and (b)), notably for $D_{d} / D_{p} \geq 7$. As in Kim's ${ }^{18}$ work, agreement between our data and $\mathrm{S} \& \mathrm{~S}^{7}$ calculations is remarkable as the binding constraint is different.
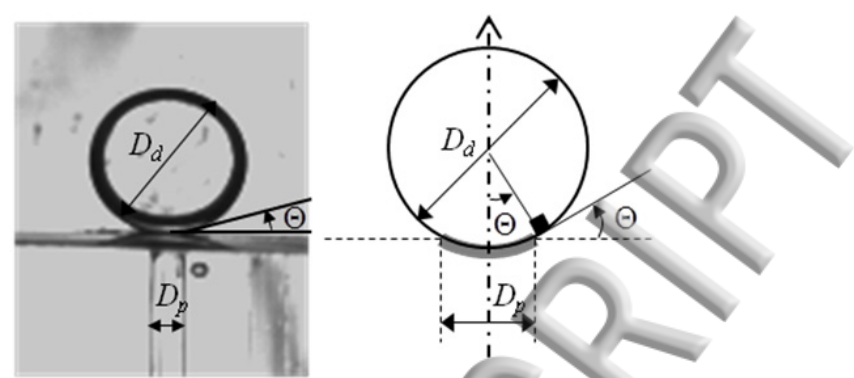

FIG. 8. Analogy between the present drop bound to a nozzle (left) and a drop in partial contact with a solid spherical cap (right) as defined by $\mathrm{S} \& \mathrm{~S}^{7,8}$.

\section{B. CRITICAL ELONGATION RATIO}

A critical elongation ratio $L_{m a x} / D_{d}$ function of the drop to pore diameter ratio leads to drop detachment ${ }^{17,19}$. We measured $L_{\max } / D_{d}$ for all parameters tested (fig. 9), $L_{\max }$ being taken from the nozzle tip to the drop apex. The points lie roughly on the same curve (fig. 9), confirming that a drop detaches in stretching mode once a critical elongation is reached. Also, we see that the critical elongation ratio is essentially a function of $D_{d} / D_{p}$.

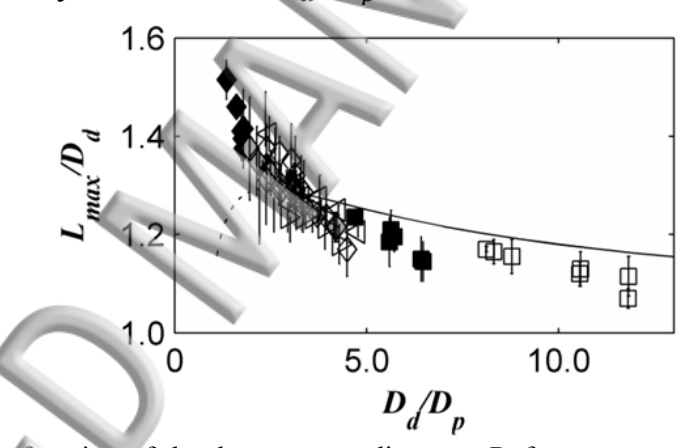

FIG. 9. Drop elongation ratio function of the drop to pore diameter. Reference system (white); system 1 (grey); system 2 (black). ( $\square) \boldsymbol{D}_{\boldsymbol{p}}=0.11 \mathrm{~mm} ;(\diamond) \boldsymbol{D}_{\boldsymbol{p}}=0.32 \mathrm{~mm}$; $\triangleleft$ ) system 3, $\boldsymbol{D}_{\boldsymbol{p}}=0.32 \mathrm{~mm}$; $(\triangleright)$ system $4, \boldsymbol{D}_{\boldsymbol{p}}=0.32 \mathrm{~mm}$. Curve from Eq. (13) (dashed and solid lines).

We remind that physicochemical properties vary for systems 1 to 4 compared to the reference system. $L_{\max } / D_{d}$ should depend on the viscosity ratio $\zeta=\eta_{d p} / \eta_{c p}$ and on the Ohnesorge number $\mathrm{Oh}=\eta_{d p} /\left[\rho_{d p} \gamma\left(D_{p} / 2\right)\right]^{1 / 2}$. The viscosity ratios we tested $(\zeta=1.5$ to 4.1$)$ may not vary enough to have-an impact on $L_{\max } / D_{d}$. For a free drop submitted to shear, Stone et al. ${ }^{28}$ found that breakup occurs above a critical elongation ratio, function of $\zeta$ but for $\zeta=0.1$ to 1 , the critical elongation ratio did not vary significantly. Similarly, we find $\mathrm{Oh}$ from $1.7 \times 10^{-2}$ to $5.3 \times 10^{-2}$. These values may be too close to observe a difference in $L_{\max } / D_{d}$, although for a free drop submitted to shear, these values are sufficiently different to obtain a twofold increase in aspect ratio. ${ }^{29}$

We return to the elongation-based criterion for stretching mode (Eq. (7)). Let us note $x_{d}^{\max }$ the displacement of the drop center of mass when the drop axial elongation is $L_{\max }$. In the limit $D_{d} \gg$ $D_{p}$, we may consider that drop deformation is entirely localized in the neck. In that case, an estimation of $x_{d}^{\max }$ is given by $\left(L_{\max }-D_{d}\right)$ and $L_{\max } / D_{d}$ roughly reads:

$$
\frac{L_{\max }}{D_{d}} \cong 1+\frac{3}{4} \lambda_{1}\left(\frac{D_{d}}{D_{p}}\right)^{-1}
$$


The curve from Eq. (13) (solid line) is plotted against our data (fig. 9). For high drop to pore diameter ratios, critical elongation ratios are well estimated by Eq. (13), in accordance with $S \& S^{7}$ or Bostwick and Steen ${ }^{10}$ : for large $D_{d} / D_{p}$, the bound drop essentially experiences a rigid motion with deformation localized at the neck. For low $D_{d} / D_{p}$, Eq. (13) is no longer valid (dashed line) and drop deformation is rather uniform. From fig. 9, we deduce that the transition from the uniform deformation regime to the localized deformation regime occurs around $D_{d} / D_{p} \cong 3$. In our setup, the neck of the bound drop preexists (without vibration) contrary to in the configuration of S\&S. ${ }^{7}$ Thus, deformation is more quickly localized (they find values for the transition in the order of 10).

\section{OSCILLATOR MODEL WITH THE TRANSIENT}

The effect of process parameters and system properties on drop diameter concord with the scaling laws of section III but threshold amplitude variations are not well predicted. Thus we developed a finer model which describes drop growth and motion as a function of time. We still consider the drop as a LFHO as it probably provides the most simple framework to study growing drop oscillations. In the moving non-inertial frame of reference where the nozzle is still, the differential equation of motion of the drop center of mass reads:

$$
\ddot{x}_{d}+2 \beta \dot{x}_{d}+\omega_{1}^{2} x_{d}=\phi \omega^{2} A \sin (\omega t+\pi)+\phi g
$$

Drop mode 1 eigenpulsation $\omega_{1}$ is given by Eq. (12) of $S \& S^{7}$ and calculated using Smithwick and Boulet's method. ${ }^{9}$ The damping coefficient $\beta$ is estimated from the empirical Eq. (4) of Bisch et al. ${ }^{6}$ We suppose drop growth is slow enough for Eq. (14) to hold at every moment. The drop diameter increases in time $t$ according to:

$$
D_{d}(t)=\left(D_{d}^{3}(0)+\frac{6}{\pi} q t\right)^{1 / 3}
$$

$\omega_{1}$ and $\beta$ depend on $D_{d}$, so vary with time as well. Equation (14) is solved numerically by the fourth order Runge-Kutta method. The integration time step is $0.01 f^{-1}$. We begin calculations with $D_{d}(0)=1.01 \times D_{p}$ as $\omega_{1}$ is not defined for $D_{d} / D_{p} \leq 1$. We fix initial conditions of $x_{d}(0)=\phi g / \omega_{1}^{2}$ and $\dot{x}_{d}(0)=0$. Figure 3 reports simulation results (dotted line) with $A$ ranging from 0 to $0.325 \mathrm{~mm}$ (increment of $0.005 \mathrm{~mm}$ ) for one dataset on the reference system (a typical drop center of mass simulated motion close to the threshold is shown in supplementary material E). Drop size at the transition is well predicted but it is overestimated far from the transition. Moreover, the threshold amplitude is underestimated: in this example, the predicted value is twice lower than experimentally.

In the example of fig. 4(a), we see that threshold values from the model (dashed lines) are well below experimental ones. Since the present model accounts for the transient (contrary to the model ${ }^{19}$ briefly reported in section III), these discrepancies cannot be attributed to the time spent by the bound drop in its resonance range as advanced earlier ${ }^{19}$. This is consistent with the experimental results of section IV.B which show that the dispersed phase flow rate little affects the threshold amplitude. The effect of the frequency on the amplitude variations is also not well predicted. Finally, the effect of the pore diameter on the threshold amplitude is opposite to experimentally. We infer that damping is underestimated in this model and the effect of $D_{d} / D_{p}$ is not well described.

\section{OSCILLATOR MODEL WITH ADDITIONAL FRICTION TERM $\boldsymbol{\beta}_{\text {film }}$}

We estimate the quality factor $Q$ by $\left(L_{\max }-D_{d}\right) / \phi A$ for bound drops at different amplitudes at $f=100 \mathrm{~Hz}$ (reference system). We find lower values than from Eq. (4) (around 3 times). Damping is higher than expected, even for amplitudes of the drop excitation force as low as $0.015 \mathrm{~mm}(9 \%$ of the drop radius, well below $\left.A_{t h}\right) \cdot Q$ is constant below the threshold amplitude $A_{t h}$, so we assume that at $A_{t h}$ and below, nonlinear effects are weak and do not explain the higher damping.

Our system undergoes additional friction compared to configurations in the literature. ${ }^{6,7,11}$ As the dispersed phase does not wet the nozzle tip, there is a wedge between the drop and nozzle surface, containing continuous liquid phase (fig. 8). Assuming $x_{d}(t) / D_{d} \ll D_{p} / D_{d} \ll 1$, an estimate of the wedge angle $\theta$ is given by $D_{p} / D_{d}$. When the drop oscillates, $\theta$ oscillates. The continuous phase in the wedge is driven outwards (inwards, resp.) when $\theta$ decreases (increases) with time. The viscous 
friction associated with the film flow leads to an extra friction term in the LFHO model of the oscillating drop. We note $\beta_{\text {film }}$ the damping coefficient associated with the friction in the film and propose the following expression (see Appendix for details):

$$
\beta_{\text {film }}=\mathcal{C} \frac{\eta_{c p}}{\rho_{d p}} \frac{D_{p}^{\alpha}}{D_{d}^{\alpha+2}}
$$

$\mathcal{C}$ is dimensionless. We infer $\mathcal{C}$ depends only on the viscosity ratio $\eta_{d p} / \eta_{c p}$ and $\alpha$ depends on the deformation regime ("uniform" or "localized"). The differential equation of motion of the drop center of mass now reads:

$$
\ddot{x}_{d}+2\left(\beta+\beta_{\text {film }}\right) \dot{x}_{d}+\omega_{1}^{2} x_{d}=\phi \omega^{2} A \sin (\omega t+\pi)+\phi g
$$

We solve Eq. (17) by the same procedure as for Eq. (14). $\alpha$ and $\mathcal{C}$ are identified from experimental threshold amplitudes since the slope of the curve $A_{t h}(f)$ is related to $\alpha$ and the curve is translated up or down by increasing or decreasing $\mathcal{C}$, respectively. $\alpha$ and $\mathcal{C}$ values are summarized in table II.

$\alpha$ was determined by fitting simulation results to the data $A_{t h}(f)$ for the reference system with $D_{p}=0.32 \mathrm{~mm}$ and $D_{p}=0.11 \mathrm{~mm}$. We needed to introduce two distinct values of $\alpha$ depending on $D_{d} / D_{p}$. For $D_{d} / D_{p} \leq 5$, the data (obtained with $D_{p}=0.32 \mathrm{~mm}$ ) are well represented with $\alpha=-1.9$.

\begin{tabular}{|c|c|c|c|}
\hline $\begin{array}{c}D_{d} / D_{p} \\
\alpha\end{array}$ & & & $\begin{array}{l}\geq 5 \\
-1.4\end{array}$ \\
\hline$\eta_{d p} / \eta_{c p}$ & 1.5 & 3.6 & 1.5 \\
\hline $\mathcal{C}$ & 4.4 & 7.4 & 8.2 \\
\hline
\end{tabular}
For $D_{d} / D_{p}>5$, the data $\left(D_{p}=0.11 \mathrm{~mm}\right)$ are better represented with $\alpha=-1.4$.

When $\alpha=-1.9$ (resp. $\alpha=-1.4$ ), the viscous force per length unit of pore circumference that acts against the drop oscillations scales as $\theta^{-2.9}$ (resp. $\theta^{-2.4}$ ). In the case where a viscous force opposes a contact line movement, the dependence on the wedge angle is weaker: the force per length unit of the contact line scales as $\theta^{-1}$. Indeed, the wedge angle is constant and the wedge translates parallel to the surface whereas in this case, the wedge angle varies, inducing the liquid flow in the wedge.

The dispersed to continuous phase viscosity ratio is of $1.5,2$ and 3.6 for the reference system, systems 3 and 4, respectively. We determined $\mathcal{C}$ for the different ratios by fitting simulation results to the data $A_{t h}(f)$ (with $\alpha$ previously identified). $\mathcal{C}$ increases monotonously from 4.4 to 7.4 when $\eta_{d p} / \eta_{c p}$ increases from 1.5 to 3.6. We logically expect that at a set $\eta_{c p}$, the viscous friction in the film increases with $\eta_{d p} / \eta_{c p}$ (drop interface becomes less and less mobile).

Figure 3 shows that the threshold amplitude is well reproduced by adding $\beta_{\text {film }}$ (solid line). Drop diameter at the transition is also well predicted. However, drop diameters are still overestimated far from the transition. Above $A_{t h}$, this may be due to nonlinear effects. A downwards shift in resonance frequency occurs when increasing $A$ for soft nonlinear oscillators. ${ }^{15,16}$ Therefore, at the set forcing frequency, smaller drops which would usually resonate at higher frequencies resonate and detach in stretching mode. ${ }^{19}$ Below $A_{t h}$, overestimation is attributed to the excitation of a higher resonance mode than mode 1. This is not taken into account in Eq. (14) or (17).

When $\beta_{\text {film }}$ is included, the effect of the frequency on amplitude variations is well described (fig. 4(a), solid lines). The effect of the pore diameter on the threshold is also well accounted for (fig. 4(a)). Below $60 \mathrm{~Hz}$, no clear threshold appeared in simulations for $D_{p}=0.11 \mathrm{~mm}$. (fig. 4(a)). We expect the drop behaves as an overdamped oscillator. Experimentally, the threshold is less sharp however exists.

We note that simulations for systems 1 and 2 were performed with $\mathcal{C}$ and $\alpha$ identified on the reference system, since the viscosity ratios are the same. Threshold amplitudes and drop diameters are relatively well predicted for these systems from the model (grey and black points, fig. 10).

Overall, the theoretical threshold amplitudes from the modified LFHO model well reproduce experimental ones (fig. 10 (a)) and drop diameters are also well accounted for (fig. 10 (b)). 

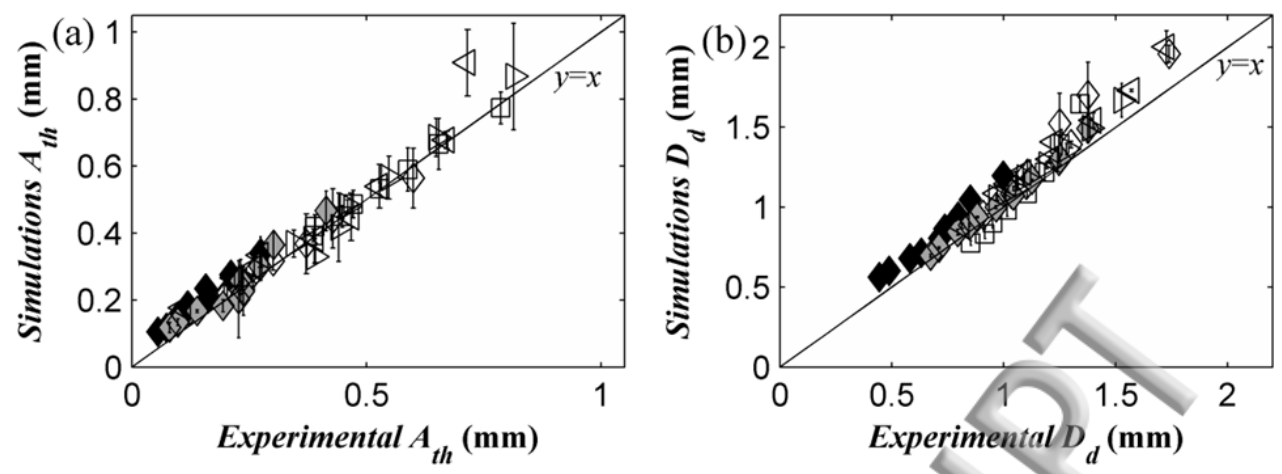

FIG. 10. Simulation and experimental values for different forcing frequencies for (a) threshold amplitudes $A_{t h}$ and (b) drop diameters at $A_{t h}$. Reference system (white); system 1 (grey); system 2 (black). ( $\square$ ) $\boldsymbol{D}_{\boldsymbol{p}}=0.11 \mathrm{~mm}$; $(\diamond) \boldsymbol{D}_{\boldsymbol{p}}=0.32 \mathrm{~mm} ;(\triangleleft)$ system $3, \boldsymbol{D}_{\boldsymbol{p}}=0.32 \mathrm{~mm} ;(\triangleright)$ system $4, \boldsymbol{D}_{\boldsymbol{p}}=0.32 \mathrm{~mm}$.

\section{CONCLUSIONS}

Studies on vibrated growing drops are rare ${ }^{19}$ compared to those on constant volume drops. ${ }^{5-18}$ In addition, few studies focus on vibrated drops where detachment oecurs from the surface. ${ }^{17-19}$ In the present work, we studied drop growth and detachment from an axially vibrating nozzle. We studied the impact of forcing parameters as well as nozzle inside diameter, dispersed phase flow rate, interfacial tension and dispersed phase viscosity. At a set forcing frequency, we observed a transition in drop diameter when increasing the forcing amplitude: above a threshold, drops detach at resonance, i.e., when the first eigenfrequency of the growing drop coincides with the forcing frequency. Below the threshold, larger drops detach in dripping mode, driven by buoyancy. The diameter of the drops formed above the threshold is very well correlated to the mode 1 eigenfrequency calculated by $S \& S^{7}$. We remind that the eigenfrequency depends on the support and drop diameters, phase densities and interfacial tension. The agreement between our results and calculations of $S \& S^{7,8}$ is remarkable as the binding constraint is different.

We examined the critical elongation ratio for drop detachment, which depends on the drop to pore diameter. We discerned two deformation regimes: for low $D_{d} / D_{p}$, a uniform deformation regime and for larger $D_{d} / D_{p}$, a localized deformation regime (limited to the neck). The neck preexists, so the latter regime appears earlier than in the configuration of $\mathrm{S} \& \mathrm{~S} .{ }^{7,8}$ We proposed a transient model to account for the threshold amplitude variations. To our knowledge, critical amplitudes for drop ejection have not been accounted for before. We modelled the growing drop as a LFHO, with the eigenfrequency of $S \& S^{7,8}$. Since the dispersed phase does not wet the nozzle, we introduced an extra damping coefficient to account for the viscous dissipation in the film of continuous phase between the drop and nozzle surface. The friction force is described as a power law of the pore to drop diameter ratio. The exponent depends on the deformation regime and the multiplier constant on the viscosity ratio. Our model well reproduces the experimental threshold amplitudes and resulting drop diameters.

In further work, it would be interesting to study drop generation when axial vibration is coupled to the shear stress exerted by a circulating phase, to approach vibrating membrane emulsification conditions.

\section{SUPPLEMENTARY MATERIAL}

See supplementary material for insight on: A, the interfacial tension at the intermediate plateau; B, the characteristic time to reach this plateau; $\mathrm{C}$, the figures for the influence of dispersed phase flow rate; $\mathrm{D}$, the figures and analysis for the influence of the continuous phase viscosity and $\mathrm{E}$, the drop center of mass motion with respect to the nozzle surface.

\section{ACKNOWLEDGEMENTS}

We thank J. Trubuil and T. Martin for their insight and work on the setup design, manufacturing and improvement.

\section{APPENDIX: ADDITIONAL FRICTION TERM $\beta_{\text {film }}$}


We consider a drop attached to the nozzle inner edge (fig. 11). We suppose that the drop diameter is large compared to the nozzle inner diameter and that the drop shape (at rest) can be approached by a spherical cap of angle $(\pi-\Theta)$. $\Theta$ is the angle of the wedge formed between the drop at rest and the nozzle surface. $\Theta$ is given by $\Theta=\arcsin \left(D_{p} / D_{d}\right) \cong D_{p} / D_{d}$.

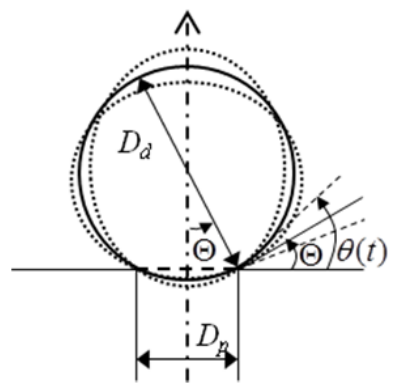

FIG. 11. Sketch of an attached drop oscillating between prolate and oblate shapes

When the drop is submitted to vibrations, we consider that it may be described by a truncated ellipsoid of revolution that oscillates between prolate and oblate shapes. The wedge angle varies with time as the drop oscillates. Its instantaneous value is $\theta(t) \cong\left(1+4 x_{d}(t) / D_{d}\right) D_{p} / D_{d}$ in the limit of small drop deformations. The continuous phase in the wedge is driven outwards (inwards, resp.) when $\theta$ decreases (increases, resp.) with time. The viscous friction associated with the film flow in the wedge leads to an additional friction term in the LFHO model of the oscillating drop. We note $F_{\text {film }}$ the corresponding friction force that acts against drop axial oscillations. Under the assumption $x_{d}(t) / D_{d} \ll D_{p} / D_{d} \ll 1$, we infer that $F_{\text {film }}$ depends on $\eta_{c p}, \eta_{d p}, D_{p}, D_{d}$ and $\dot{x}_{d}$. From dimensional arguments, we deduce:

$$
\frac{F_{\mathrm{film}}}{\eta_{c p} D_{p} \dot{x}_{d}}=\mathcal{F}\left(\frac{\eta_{d p}}{\eta_{c p}}, \frac{D_{p}}{D_{d}}\right)
$$

In the case of a viscous force that opposes a contact line movement, the vicinity of the contact line is usually described as a wedge with a well-defined dynamic contact angle. The force per length unit of the contact line is proportional to the liquid viscosity and is inversely proportional to the dynamic contact angle. In analogy to this, we seek a law in the generic form:

$$
\frac{F_{\text {film }}}{\eta_{c p} D_{p} \dot{x}_{d}}=\mathcal{G}\left(\frac{\eta_{d p}}{\eta_{c p}}\right) \times\left(\frac{D_{p}}{D_{d}}\right)^{\alpha \prime}
$$

with $D_{p} / D_{d}$ the wedge angle and $\mathcal{G}$ a function of $\eta_{d p} / \eta_{c p}$. We note that, for a moving contact line, the wedge angle is constant and the wedge translates parallel to the surface whereas in our case, the wedge angle varies, leading to the liquid flow in the wedge. We deduce the expression of the damping coefficient $\beta_{\text {film }}$ (associated with $F_{\text {film }}$ ) appearing in Eq. (17):

$$
\beta_{\mathrm{film}}=\mathcal{C}\left(\frac{\eta_{d p}}{\eta_{c p}}\right) \times \frac{\eta_{c p}}{\rho_{d p}} \frac{D_{p}^{\alpha \prime+1}}{D_{d}^{\alpha \prime+3}}=\mathcal{C}\left(\frac{\eta_{d p}}{\eta_{c p}}\right) \times \frac{\eta_{c p}}{\rho_{d p}} \frac{D_{p}^{\alpha}}{D_{d}^{\alpha+2}}
$$

where $\mathcal{C}=\mathcal{C}\left(\eta_{d p} / \eta_{c p}\right)$ depends on the dispersed to continuous phase viscosity ratio.

${ }^{1}$ C. Arnaud, European patent n ${ }^{\circ}$ EP 1551540 B1 (2006).

${ }^{2}$ E. Lepercq-Bost, Ph.D. thesis, Ecole Centrale Paris (2008).

${ }^{3}$ Lord Rayleigh, "On the Capillary Phenomena of jets,” Proc. R. Soc. Lond. 29, 71 (1879).

${ }^{4}$ H. Lamb, Hydrodynamics, 6th ed. (Cambridge University Press, Cambridge UK, 1932) p.473-475.

${ }^{5}$ H. Rodot, C. Bisch and A. Lasek, "Zero-gravity simulation of liquids in contact with a solid surface," Acta Astronaut. 6, 1083 (1979).

${ }^{6}$ C. Bisch, A. Lasek and H. Rodot, "Comportement hydrodynamique de volumes liquides sphériques semi-libres en apesanteur simulée," J.

Méc. Théor. Appl. 1 (1), 165 (1982). 
${ }^{8}$ M. Strani and F. Sabetta, "Viscous oscillations of a supported drop in an immiscible fluid," J. Fluid Mech. 189, 397 (1988).

${ }^{9}$ R. W. Smithwick III and J. A. Boulet, "Vibrations of Microscopic Mercury Droplets on Glass,” J. Colloid Interface Sci. 130 (2), 588 (1989).

${ }^{10}$ J. B. Bostwick and P. H. Steen, "Capillary oscillations of a constrained liquid drop," Phys. Fluids 21, 032108 (2009).

${ }^{11}$ J. Vejrazka, L. Vobecka and J. Tihon, “Linear oscillations of a supported bubble or drop,” Phys. Fluids 25, 062102 (2013).

${ }^{12}$ N. Abi Chebel, F. Risso and O. Masbernat, "Inertial modes of a periodically forced buoyant drop attached to a capillary,” Phys. Fluids $\mathbf{2 3}$, 102104 (2011).

${ }^{13}$ X. Noblin, A. Buguin and F. Brochard-Wyart, "Vibrated sessile drops: Transition between pinned and mobile contact line oscillations,"

Eur. Phys. J. E 14, 395 (2004)

${ }^{14}$ E. D. Wilkes and O. A. Basaran, “Forced oscillations of pendant (sessile) drops," Phys. Fluids 9, 1512 (1997).

${ }^{15}$ D. W. DePaoli, J. Q. Feng, O. A. Basaran and T. C. Scott, "Hysteresis in forced oscillations of pendant drops," Phys. Fluids 7, 1181 (1995).

${ }^{16}$ E. D. Wilkes and O. A. Basaran, "Hysteretic response of supported drops during forced oscillations," J. Fluid Mech. 393, 333 (1999).

${ }^{17}$ E. D. Wilkes and O. A. Basaran, “Drop Ejection from an Oscillating Rod,” J. Colloid Interface Sci. 242, 180 (2001).

${ }^{18}$ H. Y. Kim, "Drop fall-off from the vibrating ceiling," Phys. Fluids 16, 474 (2004).

${ }^{19}$ A. Bertrandias, H. Duval, J. Casalinho and M.-L. Giorgi, “Good vibrations - Transition in drop generation from an immersed capillary tube,” EPL 111, 44004 (2015)

${ }^{20}$ W.S. Rasband, ImageJ, U. S. National Institutes of Health, Bethesda, Maryland, USA, http://imagej.nih.gov/ij/, 1997-2014.

${ }^{21}$ C. Clanet and J.C. Lasheras, “Transition from dripping to jetting,” J. Fluid Mech. 383, 307 (1999).

${ }^{22}$ T. Tate, "On the magnitude of a drop of liquid formed under different circumstances," Phil. Mag. 27 (181), 176 (1864).

${ }^{23}$ W. D. Harkins and F. E. Brown, "The determination of surface tension (free surface energy), and the weight of falling drops: the surface tension of water and benzene by the capillary height method," J. Am. Chem. Soc. 41 (4), 499 (1919).

${ }^{24}$ Y. H. Mori, “Harkins-Brown Correction Factor for Drop Formation,” AIChE J. 36 (8), 1272 (1990).

${ }^{25}$ E. Butikov, Simulations of Oscillatory Systems (CRC Press, Taylor \& Francis Group, 2015) p.38-45.

${ }^{26}$ G. F. Scheele and B. J. Meister, "Drop Formation at Low Velocities in Liquid-Liquid Systems: Part I. Prediction of Drop Volume," AIChE J. 14 (1), 9 (1968).

${ }^{27}$ R. F. Meyer and J. C. Crocker, “Universal Dripping and Jetting in a Transverse Shear Flow,” Phys. Rev.Lett. 102, 194501 (2009).

${ }^{28}$ H. A. Stone, B. J. Bentley and L. G. Leal, “An experimental study of transient effects in the breakup of viscous drops,” J. Fluid Mech. $173,131(1986)$

${ }_{29}$ A. A. Castrejon-Pita, J. R. Castrajon-Pita and I. M. Hutchings, “Breakup of Liquid Filaments,” Phys. Rev. Lett. 108, 074506 (2012).

${ }^{30}$ A. Bonfillon and D. Langevin, "Viscoelasticity of Monolayers at Oil-Water Interfaces," Langmuir 9, 2172 (1993).

${ }^{31}$ S. R. Deshiikan, D. Bush, E. Eschenazi and K. D. Papadopoulos, "SDS, Brij58 and CTAB at the dodecane-water interface," Colloids and Surfaces A: Physicochem. Eng. Aspects 136, 133 (1998).

${ }^{32}$ A. Bonfillon, F. Sicoli and D. Langevin, “Dynamic surface tension of ionic surfactant solutions," J. Colloid Interface Sci. 168, 497 (1994). 
${ }^{33}$ B. Lindman, M.-C. Puyal, N. Kamenka, R. Rymden and P. Stilbs, "Tracer self-diffusion studies of micelle formation of a short-chain

ionic surfactant, sodium n-octanoate," J. Phys. Chem. 88 (1), 5048 (1984).

${ }^{34}$ H. G. Gomaa, J. Liu and J. Zhu, "Experimental and theoretical analysis of emulsification characteristicsusing a high porosity microscreen under oscillatory shear conditions," Colloids and Surfaces A: Physicochem. Eng. Aspects 456, 160 (2014).

${ }^{35}$ R. Clift, J. Grace and M. E. Weber, Bubbles, Drops and Particles (Dover Publications, Inc., 2005) p.191-197.

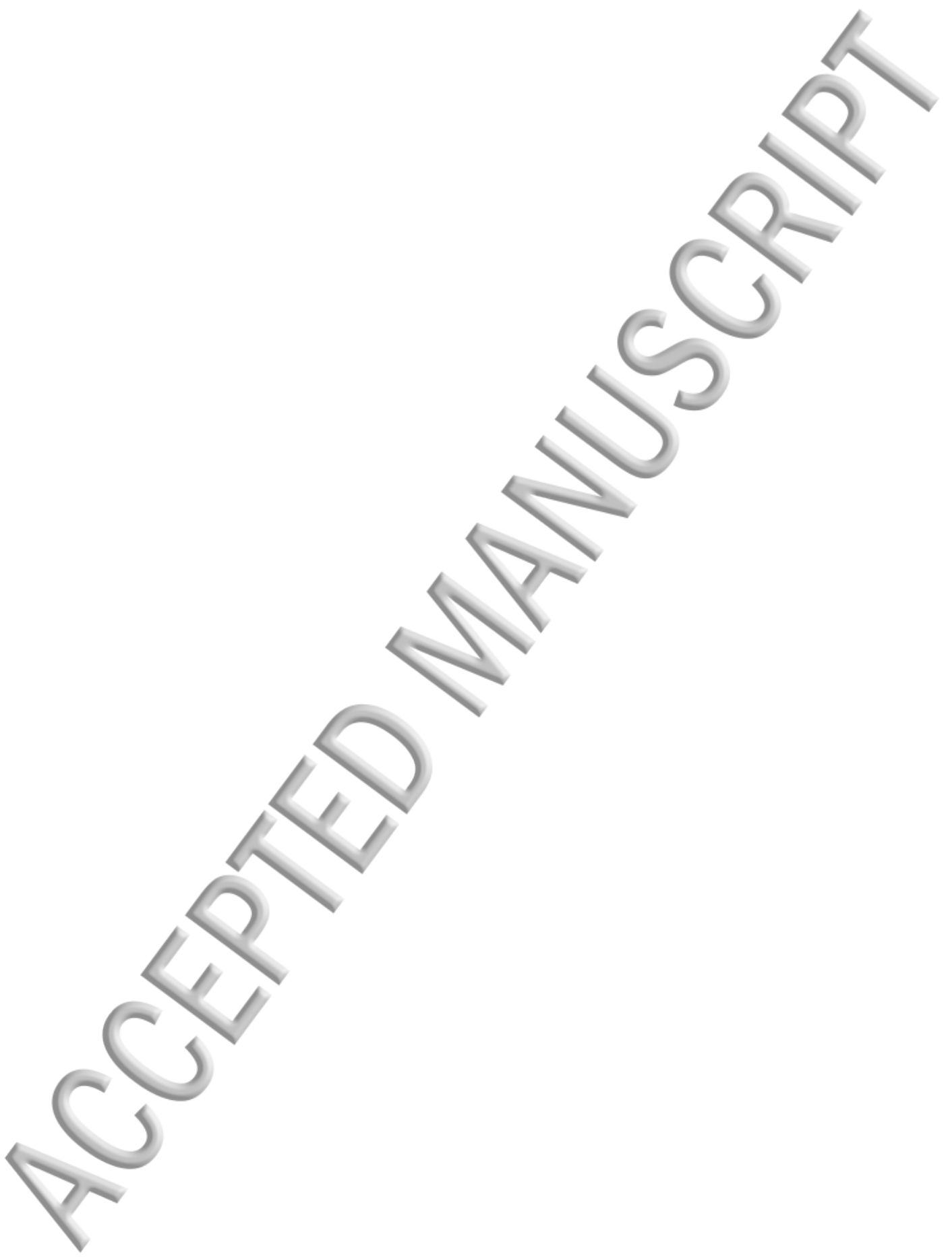

